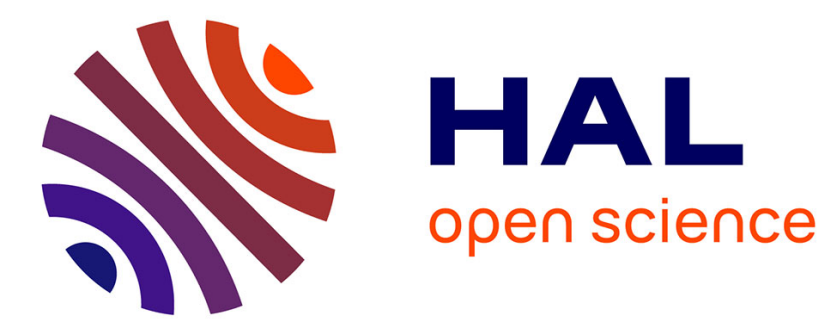

\title{
Stylet inscrit de Rouffach (Haut-Rhin)
}

Michel Feugère

\section{To cite this version:}

Michel Feugère. Stylet inscrit de Rouffach (Haut-Rhin). Gallia - Archéologie de la France antique, 2000, 57, pp.227-229. 10.3406/galia.2000.3020 . hal-01901993

\section{HAL Id: hal-01901993 \\ https://hal.science/hal-01901993}

Submitted on 16 Jan 2020

HAL is a multi-disciplinary open access archive for the deposit and dissemination of scientific research documents, whether they are published or not. The documents may come from teaching and research institutions in France or abroad, or from public or private research centers.
L'archive ouverte pluridisciplinaire $\mathbf{H A L}$, est destinée au dépôt et à la diffusion de documents scientifiques de niveau recherche, publiés ou non, émanant des établissements d'enseignement et de recherche français ou étrangers, des laboratoires publics ou privés.

\section{(ㅇ)(1) $\$$}

Distributed under a Creative Commons Attribution - NonCommercial - NoDerivatives 44.0 


\title{
STYLET INSCRIT \\ DE RoufFaCH (HAUT-RHIN)
}

\author{
Michel FEUGÈRE*
}

Mots-clés. Stylet, inscription, aphorisme, ars amandi.

Key-words. Stylus, inscription, aphorism, ars amandi,

Résumé. Ie stylet en bronze de Rouffach comporte, répartie sur quatre faces, une inscription énonsant un aphorisme : amori / ars mea / cum studio / procedet, dont le sens peut se comprendre au premier degré, les lettres écrites avec ce stylet pouvant faire naître et progresser l'amour. Mais la possibilité d'une lecture érotique, au deuxième degré, révèle sans doute le raffinement de conversations galantes en usage dans la bonne sociélé gallo-romaine.

\begin{abstract}
The bronze stylus of Rouffach bears an inscription extending over its four sides setting forth an aphorism : amori / ars mea / cum studio / procedet that could, at first, be taken to mean that letters writlen with this stylus could awaken love and make it thrive. But on second reading, there is the possibility of a more erotic interpretation that, without a doubt, reveals the refined language of courting in usage by upper Gallo-Roman society.
\end{abstract}

Au cours des fouilles effectuées en 1979 à Rouffach (Haut-Rhin) par P. Faust, un stylet en bronze a été recueilli dans une berme. Cette trouvaille, pour ainsi dire fortuite, n'a pas permis d'associer l'objet à l'un ou l'autre des niveaux archéologiques mis en évidence à cette occasion, et cette intéressante découverte ne peut être datée que par référence à l'occupation du site qui concerne principalement les deux premiers siècles de notre ère ${ }^{l}$.

\section{DESCRIPTION}

L'objet se présente sous la forme d'une courte tige en bronze, coulée d'un seul tenant, dont la faible taille (90 $\mathrm{mm}$ ) surprend quelque peu (fig. 1); bien que nous

\footnotetext{
* UMR 154 du CNRS, 390 avenue de Pérols, F-34530 Lattes.

1. Voir les informations archéologiques de Gallia, 36, 2, 1978, p. $354-355$ et $38,2,1980$, p. $443-444$ où il est fait mention de ce stylet et de son inscription.
}

soyons habitués à écrire avec des instruments plus allongés, cette longueur suffit cependant pour que l'objet, placé entre le pouce et l'index d'une main adulte, repose sur le côté de la paume. Il n'est donc pas nécessaire de supposer qu'on est en présence d'un ustensile destiné à un enfant, encore que cette interprétation ne puisse être écartée a priori.

Le stylet comporte tout d'abord une pointe fonctionnelle, longue de $13 \mathrm{~mm}$, qui peut avoir été raccourcie par des affûtages successifs; on observe en effet sur les stylets de forme analogue une pointe sensiblement plus longue. Cette fine tige lisse est interrompue par une série de moulures qui, sur tous ces instruments, marque le début de la zone destinée à assurer la prise en main. Ces moulures se prolongent ici par un segment lisse, de section carrée, long de $24 \mathrm{~mm}$. Sur chaque face, deux petites encoches triangulaires dégagent aux extrémités des queues d'arondes, transformant le côté de la tige en tabula ansata. C'est cette surface ainsi préparée qui a reçu une inscription répartie sur les quatre faces du stylet. 

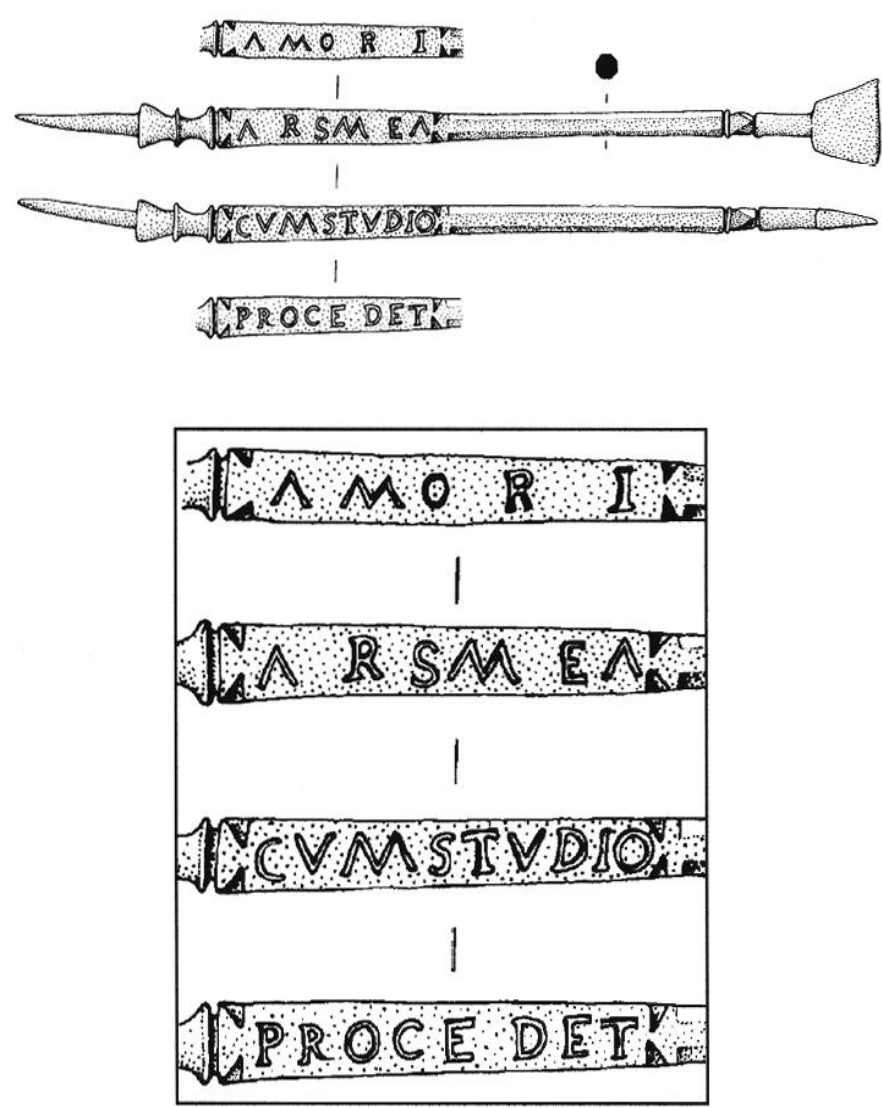

Fig. 1 - Stylet inscrit de Rouffach (échelle: $1 / 1$ ) et détail de l'inscription (échelle : $x 2$ ).

\section{TYPOLOGIE}

Les stylets gallo-romains affectent des formes variées, mais l'examen des caractères communs permet de distinguer plusieurs variantes. Tous possèdent quatre parties distinctes, plus ou moins développées selon les types: une pointe généralement de faible diamètre, mêmc dans sa partic la plus ćpaissc ; unc zone moulurée destinée à faciliter la préhension ; une tige normalement lisse ; une palette de forme usuellement trapézoïdale.

Malgré la corrosion qui en masque souvent le détail, les stylets romains en fer sont souvent des objets très soigneusement décorés, comme quelques objets lisibles ${ }^{2}$ ou de nouvelles techniques de nettoyage permettent

2. Par exemple certains stylets de Chassey-lès-Montbozon, cf. M. Feugère, Les petits objets, in: G. Barbet, P. Gandel (éds), Chasseylès-Montbozon. Un établissement rural gallo-romain, Annales littéraires de l'université de Besançon, 627, Paris, 1997, 166 sqq., $\mathrm{n}^{\text {os }} 68$ et 1559-1561. désormais de le découvrir ${ }^{3}$. Des travaux en cours devraient permettre de préciser l'évolution morphologique, encore très mal connue, des stylets au cours de l'Antiquité romaine (recherches de V. Schaltenbrand Obrecht) ${ }^{4}$.

\section{INSCRIPTION}

Les lettres composant l'inscription sont profondément gravées en capitales et pourvues de légers empattements. Bien que chaque caractère soit parfaitement formé, le graveur a éprouvé les plus grandes difficultés à répartir correctement les lettres et les espaces, comme il arrive fréquemment, même sur les inscriptions lapidaires. On lit sans aucune incertitude : amori / ars mea / cum studio / procedet.

L'inscription se présente donc sous la forme d'un aphorisme, commodément réparti en quatre mots ou groupes de mots tenant sur chacune des faces du stylet. Elle forme une phrase complète dont le verbe, procedet, se place normalement en latin à la fin, mais d'autres constructions sont possibles selon l'importance que l'on souhaite donner à l'un ou l'autre membre de la phrase ; ces constructions différentes, on le verra, modifient sensiblement le sens de l'ensemble.

Procedere signifie normalement s'avancer, aller de l'avant, au sens propre mais également au sens figuré de " faire des progrès ". À ce sens premier s'ajoute plus subtilement l'idée d'arriver à tel ou tel succès, mais aussi de manière plus ouverte arriver à tel ou tel résultat. Le sujet de ce verbe est évidemment ars mea : ars possède en latin un sens technique, aujourd'hui vieilli, de talent, savoir-faire. Studium a enfin le sens d'application, étude, pratique, mais signifie plus couramment l'application zélée, l'ardeur.

L'inscription peut donc se traduire mot à mot : « mon savoir-faire fait progresser l'amour grâce à mon zèle ", ce qui se comprend bien si le stylet parle à son utilisateur : " grâceà moi, aux lettres que j'écris, tu peux progresser

3. Voir Schaltenbrand Obrecht, Wie wurden eiserne stili in römischer Zeit hergestellt und verziert ?, in: R. Ebersbach, A. R. Furger (éds), Mille Fiori Festschrift L. Berger, Forsch. Augst 25, 1998, p. 201-205.

4. En attendant, l'un des rares essais de classification des stylets en fer est la tentative élaborée à partir de l'abondante série de Javols (Lozère) : P. Peyre, avec la collab. de J.-C. Béal et J. Sirvin, Javols (fouilles 1969-1979). Les objets de fer, Mende, 1979, p. 29-54. 
en amour ". Dans une intéressante étude publiée en 1994, G. E. Thüry a remarqué que plusieurs inscriptions gravées sur des petits objets d'usage quotidien, dont ce stylet, portaient des inscriptions selon lui volontairement équivoques ${ }^{5}$. Ainsi l'aphorisme du stylet de Rouffach peut-il aussi être compris de la manière suivante: " mon amour bénéficie de mon savoir-faire ", avec amor désignant par métonymie la personne aimée. Ars devient dans ce cas ars [amandi], la technique érotique.

Il est extrêmement rare que des stylets portent une inscription, puisqu'on connaît à ce jour moins d'une dizaine d'objets présentant cette particularité. Pour les stylets inscrits sur quatre faces, comme celui de Rouffach, on peut mentionner les découvertes de Volon en HauteSaône (utere / felix / digne / merito) ${ }^{6}$, de Cologne (hego / scribo / sinem / manum), de Frankfurt-Heddernheim (dicta / felix / felicior / scribe), du musée Rolin à Autun (vi / ve / de / o ) ${ }^{7}$, auxquels s'ajoute désormais une trouvaille récente du Landeron, dans le Valais ( $d i$ te / servent / amor / amorum $)^{8}$. Dans le cas de Rouffach, le texte renvoie au premier degré à l'utilisation de l'objet, et

5. Cf. G. E. Thüry, Mehrdeutige erotische Kleininschriften, Bayerische Vorgeschichtsblätter, 59, 1994, p. 5-95.

6. Cf. Héron de Villefosse, communication au Bulletin archéologique du Comité des travaux historiques, 1918, p. XLV-XLVIII.

7. Tous ces exemplaires sont signalés, avec d'autres plus tardifs, par H. I.eclercq, s.v. Style, in: F. Cabrol, H. Leclercq, Dictionnaire d'archéologie chrétienne et de liturgie, 15, Paris, 1953, p. 1694-1697.

8. Cf. P. Hofmann Rognon, Un stylet en bronze dédicacé au Landeron (Suisse, VS), Bulletin Instrumentum, 8, déc. 1998, p. 26-27. éventuellement à un second degré à une activité amoureuse dont l'auteur serait évidemment le propriétaire du stylet. Mal connus dans la littérature antique conservée sur tablettes, les textes amoureux sont particulièrement bien représentés dans les graffites, notamment ceux que l'on traçait, sans aucun doute à l'aide d'un stylet, sur les enduits peints des maisons romaines. Beaucoup de ces " textes » sont des exhortations grossières ou des vantardises de noceurs ${ }^{9}$, mais l'inscription du stylet de Rouffach se place à un autre niveau. En jouant sur les différents niveaux de lecture ${ }^{10}$, cette inscription lève un voile sur les jeux de l'esprit qui pouvaient être en usage dans une certaine société gallo-romaine, où l'emploi quotidien de la rhétorique pouvait teinter de galanterie les relations sociales.

\section{Remerciements}

Je remercie chaleureusement $P$. Faust d'avoir bien voulu me confier ce stylet pour étude et d'en avoir autorisé la publication.

9. Voir la présentation commode, classée par thèmes, qu'a récemment donnée A. Varone des graffites pompéiens : Erotica Pompeiana. Iscrizioni d'amore sui muri di Pompei, Studia archaeologica, 71, Rome, 1994 ; pour la Gaule, entre autres, un exemple récent a été savamment analysé par J.-P. Bost : Exploits amoureux à Limoges au III' siècle après J.-C. : trois graffiti de la "Maison des Nones de Mars ", Travaux d'archéologie limousine, 13, 1993, p. 53-57.

10. Un cas assez semblable est fourni par une fusaiole de Trèves ( $C I L$ XIII, 10019, 17) inscrite imple me / sic versa me: cf. Thüry, p. 88-89, cité note 5 . 